\title{
Plattformen für Profis
}

Das Prinzip der Online-Plattform ist heute allgegenwärtig. Als Endverbraucher nutzen wir etwa Amazon, Ebay oder Airbnb, um bequem auf Angebote und Dienstleistungen unterschiedlicher Anbieter zugreifen zu können. Aber auch im Unternehmensumfeld spielen plattformbasierte Geschäftsmodelle eine wachsende Rolle.

Das hat auch mit dem Internet der Dinge (IoT) zu tun. Miteinander kommunizierende Gegenstände ermöglichen es etwa Wartungsdienste von überall her anzubieten. So können Anbieter und Kunden zusammenfinden, bei denen das früher zum Beispiel aus räumlichen Gründen nicht möglich war. Zurzeit nutzen aktuellen Umfragen zufolge etwa die Hälfte der Unternehmen digitale Plattformen. Bei denen, die es nicht tun fehlt in der Regel das nötige Know-how und nicht so sehr der Wille, die neuen Möglichkeiten zu erproben.

Grund genug für Wirtschaftsinformatik \& Management dem Thema einen Schwerpunkt zu widmen und so hoffentlich dazu beizutragen, einige Wissenslücken zu schließen. Die Artikel zu diesem Thema drehen sich um Mechanismen zur Gestaltung erfolgreicher digitaler Plattformen, Strategien zur Etablierung digitaler Plattformen in der Industrie, der Frage, wie man von der Erkenntnis, dass digitale Plattformen sinnvoll sind, zur entsprechenden Umsetzung gelangt oder wie der nötige Datenschutz bei der Nutzung gewährleistet werden kann. Ein weites Feld also, in dem Sie hoffentlich viel Anregendes finden und einige Aha-Momente haben werden.

Eine erkenntnisreiche Lektüre wünscht

Peter Pagel

Chefredakteur

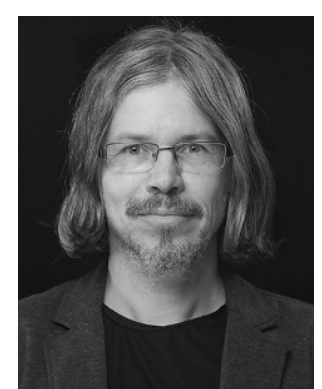

Peter Pagel $(\square)$

peter.pagel@springer.com

Wiesbaden, Deutschland 